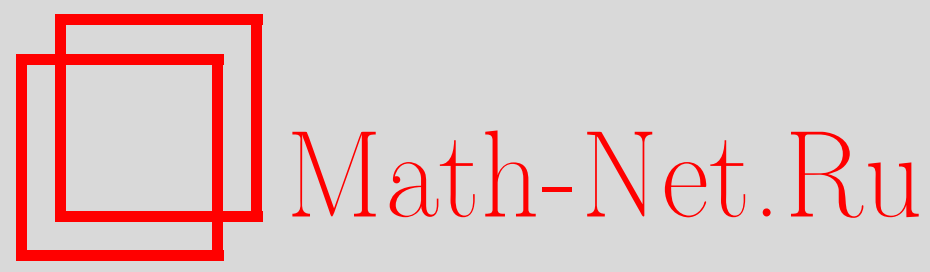

А. П. Старовойтов, О совпадении наименыших равномерных уклонений функции от полиномов и рациональных дробей, Матем. заметки, 2003, том 74, выпуск 4, 612-617

DOI: https://doi.org/10.4213/mzm295

Использование Общероссийского математического портала Math-Net.Ru подразумевает, что вы прочитали и согласны с пользовательским соглашением http://www.mathnet.ru/rus/agreement

Параметры загрузки:

IP: 54.84 .234 .179

26 апреля 2023 г., 18:03:25

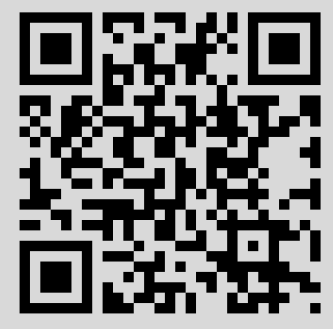




\section{О СОВПАДЕНИИ НАИМЕНЬШИХ РАВНОМЕРНЫХ УКЛОНЕНИЙ ФУНКЦИИ ОТ ПОЛИНОМОВ И РАЦИОНАЛЬНЫХ ДРОБЕЙ}

\section{А. П. Старовойтов}

Для заданной невозрастающей последовательности $\left\{a_{n}\right\}_{n=0}^{\infty}$ неотрицательных действительных чисел, которая стремится к нулю, найдены необходимые и достаточные условия на последовательность $\left\{n_{k}\right\}_{k=0}^{\infty}$, для которой существует непрерывная на отрезке [0,1] функция $f$ такая, что $R_{n_{k}, m_{k}}(f)=E_{n_{k}}(f)=a_{n_{k}}, k=0,1,2, \ldots$, где $E_{n}(f)$ и $R_{n, m}(f)$ - наилучшие равномерные приближения функции $f$ полиномами степени не выше $n$ и рациональными функциями вида $r_{n, m}(x)=p_{n}(x) / q_{m}(x)$ соответственно.

Библиография: 10 названий.

1. Постановка задачи и формулировка основных результатов. Пусть $C[a, b]$ - банахово пространство непрерьвных на отрезке $[a, b]$ вещественнозначных функций с равномерной нормой. Обозначим через $\mathscr{R}_{m}^{n}$ множество всех рациональных функций с вешественными коэффициентами, степень числителя которых не вьше $n$, а степень знаменателя не вьше $m$. В частности, $\mathscr{P}_{n}=\mathscr{R}_{0}^{n}$ и $\mathscr{R}_{n}=\mathscr{R}_{n}^{n}-$ соответственно множества всех полиномов и рациональных функций степени не выше $n$. Для функции $f \in C[a, b]$ рассмотрим ее наименьшие равномерные уклонения от рациональных дробей из $\mathscr{R}_{m}^{n}$, т.e.

$$
R_{n, m}=R_{n, m}(f)=\inf \left\{\|f-r\|: r \in \mathscr{R}_{m}^{n}\right\},
$$

где $\|g\|=\max \{|f(x)|: x \in[a, b]\}$. Тогда величины

$$
E_{n}=E_{n}(f)=R_{n, 0}(f) \text { и } R_{n}=R_{n}(f)=R_{n, n}(f)
$$

являются соответственно наименьшими уклонениями функции $f$ от полиномов и рациональных дробей степени не выше $n$. Поскольку очевидны включения

$$
\mathscr{P}_{n+1} \supset \mathscr{P}_{n}, \quad \mathscr{R}_{m}^{n+1} \supset \mathscr{R}_{m}^{n}, \quad \mathscr{R}_{m+1}^{n} \supset \mathscr{R}_{m}^{n}
$$

то из свойств точной нижней грани получим, что

$$
0 \leqslant R_{n+1} \leqslant R_{n} \leqslant R_{n, m+1} \leqslant R_{n, m} \leqslant E_{n} \leqslant E_{n-1}
$$

при $m=0,1, \ldots, n-1 ; n=1,2,3, \ldots$.

В силу теоремы Вейерштрасса отсюда следует, что последовательность $\left\{E_{n}(f)\right\}_{n=0}^{\infty}$ стремится к нулю, не возрастая. Отмеченные свойства наименьших полиномиальных уклонений характеризуют их полностью. Именно, справедлива следующая 
ТЕОРЕМА БЕРНШТЕЙНА [1]. Если невозрастающая последовательность $\left\{a_{n}\right\}_{n=0}^{\infty}$ неотрицательных действительных чисел сходится к нулю, то существует функиия $f \in C[a, b]$, для которой

$$
E_{n}(f)=a_{n}, \quad n=0,1,2, \ldots
$$

Аналог этой теоремы для рациональных приближений получен лишь частично. Именно, для любой невозрастающей и сходящейся к нулю последовательности действительных чисел $\left\{a_{n}\right\}_{n=0}^{\infty}$ Долженко [2] построил такую непрерывную функцию $f$, для которой при $n_{k}=9^{k}$ справедливы равенства

$$
R_{n_{k}}(f)=E_{n_{k}}(f)=a_{n_{k}}, \quad k=0,1,2, \ldots
$$

В связи с этим возник вопрос, насколько плотной в $\mathbb{N}$ может быть последовательность $\left\{n_{k}\right\}$, для которой при любом выборе последовательности $\left\{a_{n}\right\}_{n=0}^{\infty}$ с указанньги выше свойствами найдется функция $f \in C[a, b]$, удовлетворяюшая равенствам (1)?

Боэм [3] показал, что если равенства $R_{n}(f)=E_{n}(f)$ выполняются при всех $n=$ $0,1,2, \ldots$, то $f(x)=A T_{k}(x)+B$, где $T_{k}$ - некоторьй многочлен Чебьшева для отрезка $[a, b]$, а $A, B$ - постоянные. Это означает, что последовательность $\left\{n_{k}\right\}$ из (1) является некоторым собственным подмножеством $\mathbb{N}$. Позже было установлено $[4$, гл. $7, \S 2$, равенство (2.12)], что с помошью метода Долженко последовательность $n_{k}=9^{k}$ в (1) можно заменить на $n_{k}=3^{k}$. Окончательный результат был получен Пекарским [5]. Именно, последовательность $n_{k}=2^{k}-1$ является самой "медленной последовательностью", для которой имеют место равенства (1).

В данной работе рассматривается следующая

ЗАДА ЧА. Насколько плотной в $\mathbb{N}$ может быть последовательность $\left\{n_{k}\right\}$, для которой при любом выборе последовательности $\left\{a_{n}\right\}_{n=0}^{\infty}$ с указанньми вьше свойствами найдется функция $f \in C[a, b]$, удовлетворяюшая равенствам

$$
R_{n_{k}, m_{k}}(f)=E_{n_{k}}(f)=a_{n_{k}}, \quad k=0,1,2, \ldots ?
$$

Ответ на поставленный вопрос дают следуюшие теоремы 1 и 2.

ТЕОРема 1. Пусть последовательности $\left\{n_{k}\right\}_{k=0}^{\infty},\left\{m_{k}\right\}_{k=0}^{\infty}$ неотричательных чельх чисел удовлетворяют условию

$$
n_{k+1} \geqslant n_{k}+m_{k}+1, \quad k=0,1,2, \ldots
$$

Тогда существует такая функиия $f \in C[a, b]$, для которой

$$
R_{n_{k}, m_{k}}(f)=E_{n_{k}}(f)=a_{n_{k}}, \quad k=0,1,2, \ldots
$$


ТЕОрема 2. Пусть для функиии $f \in C[a, b]$ и некоторых наборов $\left\{m_{j}\right\}_{j=0}^{k}$, $\left\{n_{j}\right\}_{j=0}^{k}$ чельх неотрицательных чисел $0 \leqslant n_{0}<n_{1}<\cdots<n_{k}$ имеют место соотношения:

$$
\begin{gathered}
E_{n_{0}}>E_{n_{1}}>\cdots>E_{n_{k}}, \\
R_{n_{j}, m_{j}}=E_{n_{j}}, \quad j=0,1, \ldots, k .
\end{gathered}
$$

Тогда найдется набор $\left\{n_{j}^{\prime}\right\}_{j=0}^{k}$ таких чельх неотричательных чисел, что $n_{j}^{\prime} \leqslant n_{j}$ $u$

$$
n_{j+1}^{\prime} \geqslant n_{j}^{\prime}+m_{j}+1 \quad n p u \quad j=0,1,2, \ldots, k .
$$

Положив в теоремах 1 и $2 m_{k}=n_{k}, k=0,1,2, \ldots$, получим упомянутый вьше результат Пекарского.

СЛЕДСТВИЕ 1 [5]. Для последовательности $n_{k}=2^{k}-1$ существует непрерывная на отрезке $[a, b]$ функиия $f$, для которой выполняются соотношения (1). Обратно, если для функиии $f \in C[a, b]$ и некоторого набора челых неотричательных чисел $0 \leqslant n_{0}<n_{1}<\cdots<n_{k}$ выполняются соотношения

$$
E_{n_{0}}>E_{n_{1}}>\cdots>E_{n_{k}}, \quad R_{n_{j}}=E_{n_{j}}, \quad j=0,1,2, \ldots, k
$$

mo $n_{k} \geqslant 2^{k}-1$.

Рассмотрим случай, когда $m_{k}=m, k=0,1,2, \ldots$, где $m$ - фиксированное целое неотрицательное число.

СлЕДСТВИЕ 2. Для любого фиксированного целого неотрицательного числа $m$ существует такая функиия $f \in C[a, b]$, что

$$
R_{(m+1) k, m}=E_{(m+1) k}=a_{(m+1) k}, \quad k=0,1,2, \ldots
$$

В частности, при $m=1$ найдется такая функиия $f \in C[a, b]$, что

$$
R_{2 k, 1}=E_{2 k}=a_{2 k}, \quad k=0,1,2, \ldots
$$

Отметим, что ранее Назаренко [6] построил пример функции $f$ из класса Харди $H_{2}$, для которой имеют место левые равенства (5) в случае наименњших уклонений $f$ от рациональных дробей и полиномов в метрике пространства $H_{2}$.

СлЕДСТВИЕ 3. Если $f$ непрерывна на отрезке $[a, b]$ и для некоторого набора иелых чисел $0 \leqslant n_{0}<n_{1}<\cdots<n_{k}$ выполняются соотношения

$$
E_{n_{0}}>E_{n_{1}}>\cdots>E_{n_{k}}, \quad R_{n_{j}, m}=E_{n_{j}}, \quad j=0,1, \ldots, k
$$

mo $n_{k} \geqslant(m+1) k$.

Таким образом, когда рассматриваются наилучшие диагональные рациональные приближения, самая "медленная последовательность" $\left\{n_{k}\right\}$, удовлетворяющая (2) вместе с некоторой функцией $f \in C[a, b]$, растет с точностью до константы как геометрическая прогрессия $\left\{2^{k}\right\}_{k=0}^{\infty}$. В случае $m$-й строки ее рост совпадает с ростом арифметической прогрессии $\{(m+1) k\}_{k=0}^{\infty}$. 
2. Доказательство основных результатов. Перейдем к доказательству теорем 1 и 2.

ДОКАЗАТЕЛЬСТВО ТЕОРЕМЫ 1. Введем вспомогательную последовательность $\left\{a_{n}^{\prime}\right\}_{n=0}^{\infty}$ :

$$
a_{n}^{\prime}=\left\{\begin{array}{l}
a_{n}, \quad \text { если } n=0,1,2, \ldots, n_{0}-1 \\
a_{n_{k}}, \quad \text { если } n=n_{k}, n_{k}+1, \ldots, n_{k+1}-1, k=0,1,2, \ldots
\end{array}\right.
$$

Из теоремы Бернштейна следует существование функции $f$, для которой $E_{n}=a_{n}^{\prime}$ при $n=0,1,2, \ldots$ Покажем, что эта функция является искомой, т.е.

$$
R_{n_{k}, m_{k}}(f)=E_{n_{k}}(f)=a_{n_{k}} \quad \text { при } \quad k=0,1,2, \ldots
$$

Действительно, пусть $p_{n_{k}} \in \mathscr{P}_{n_{k}}$ есть многочлен наилучшего приближения функции $f$. Поскольку

$$
E_{n_{k}}=E_{n_{k}+1}=\cdots=E_{n_{k+1}-1}=a_{n_{k}},
$$

то $p_{n_{k}}$ будет элементом наилучшего приближения и в $\mathscr{P}_{n_{k+1}-1}$. Ввиду теоремы Чебышева разность $f-p_{n_{k}}$ имеет на $[a, b]$ по крайней мере $\left(n_{k+1}-1\right)+2=n_{k+1}+1$ точек альтернанса. Далее, применим теорему Ахиезера [7], которая дает критерий дроби наилучшего приближения. Именно, $p_{n_{k}}$ будет элементом наилучшего приближения в $\mathscr{R}_{m_{k}}^{n_{k}}$, если разность $f-p_{n_{k}}$ имеет по крайней мере $n_{k}+m_{k}+2$ точек альтернанса. Нужное количество точек имеется, поскольку из неравенства $n_{k+1} \geqslant n_{k}+m_{k}+1$ следует, что $n_{k+1}+1 \geqslant n_{k}+m_{k}+2$. Отсюда

$$
E_{n_{k}}=\left\|f-p_{n_{k}}\right\|=R_{n_{k}, m_{k}}=a_{n_{k}}, \quad k=0,1,2, \ldots
$$

Теорема доказана.

С помощью рассуждений, применяемых при доказательстве теоремы 1 , легко получить следующее следствие.

СлЕДСТВИЕ 4. Для любого фиксированного иелого неотрицательного числа $n$ и любого сколь угодно большого натурального $m$ существует такая функиия $f \in$ $C[a, b]$, для которой

$$
R_{n, m}(f)=E_{n}(f) .
$$

В частности, для $n=0$ при сколь угодно большом $m$ существует такая функиия $f \in C[a, b]$, для которой

$$
R_{0, m}(f)=E_{0}(f)
$$

ДокАЗАТЕЛЬСТВо. Для доказательства достаточно положить

$$
a_{k}^{\prime}= \begin{cases}a_{k}, & \text { если } k=0,1,2, \ldots, n-1, \\ a_{n}, & \text { если } k=n, n+1, \ldots, n+m, \\ a_{n+k}, & \text { если } k=n+m+1, n+m+2, \ldots,\end{cases}
$$

а затем воспользоваться теоремой Бернштейна. 
Следствие 4 показывает, что, во-первых, для некоторых функиий из пространства $C[a, b]$ аппроксимация дробями вида

$$
\left\{\frac{1}{p_{m}(x)}: p_{m} \in \mathscr{P}_{m}\right\}
$$

не әффективна, во-вторых, расширение множества аппроксимирующих функций c $\mathscr{P}_{n}$ до $\mathscr{R}_{m}^{n}$ для любого сколь угодно большого $m$ не дает выигрыша в скорости аппроксимации на всем пространстве $C[a, b]$. В случае, когда $n=m$, данное явление в поведении наименьших уклонений функций из $C[a, b]$ при приближении множествами $\mathscr{P}_{n}$ и $\mathscr{R}_{n}$ было обнаружено и детально исследовано Гончаром и Долженко (см., например, [2], [8]).

Следующее утверждение для приближений в $L_{p}$ получено в [9]. В более общей ситуации оно встречается в [6].

Лемма. Пусть функиия $f$ непрерывна на отрезке $[a, b]$ и для некоторых натуральных чисел $n$ и $m R_{n, m}=E_{n}<E_{n-1}$. Тогда

$$
E_{n}=E_{n+1}=\cdots=E_{n+m}
$$

ДокАЗАТЕЛьСтво. Пусть $p_{k} \in \mathscr{P}_{k}$ - элемент наилучшего приближения $f$. Так как $E_{n}<E_{n-1}$, то $\operatorname{deg} p_{n}=n$ и $n \leqslant \operatorname{deg} p_{n+m} \leqslant n+m$. Обозначим соответственно через $\varphi$ и $\psi$ остаток и частное при делении многочлена $p_{n+m}-p_{n}$ на многочлен $p_{n}$. Тогда $\varphi \in \mathscr{P}_{n-1}, \psi \in \mathscr{P}_{m}$ и

$$
(\psi+1) p_{n}+\varphi=p_{n+m} .
$$

Для достаточно малых $\lambda>0$ рассмотрим функцию

$$
r_{n, m}=\frac{p_{n}+\lambda \varphi}{1-\lambda \psi}
$$

принадлежащую множеству $\mathscr{R}_{m}^{n}$. Из (6) при $\lambda \rightarrow 0$ вытекают равенства

$$
\begin{aligned}
r_{n, m} & =\left(p_{n}+\lambda \varphi\right)(1+\lambda \psi+o(\lambda)) \\
& =(1-\lambda) p_{n}+\lambda\left[(\psi+1) p_{n}+\varphi\right]+o(\lambda) \\
& =(1-\lambda) p_{n}+\lambda p_{n+m}+o(\lambda)
\end{aligned}
$$

Следовательно,

$$
\begin{aligned}
R_{n, m}(f) & \leqslant\left\|f-r_{n, m}\right\| \leqslant(1-\lambda)\left\|f-p_{n}\right\|+\lambda\left\|f-p_{n+m}\right\|+o(\lambda) \\
& \leqslant E_{n}-\lambda\left(E_{n}-E_{n+m}\right)+o(\lambda) .
\end{aligned}
$$

Если теперь $E_{n+m}<E_{n}$, то из последнего неравенства получим, что $R_{n, m}<E_{n}$. Это противоречит условию. Лемма доказана. 
ДоКАЗАТЕЛЬСТВО ТЕОРЕМЫ 2. Построим набор таких целых неотрицательных чисел $n_{j}^{\prime} \leqslant n_{j}, n_{0}^{\prime}<n_{1}^{\prime}<\cdots<n_{k}^{\prime}$, которые удовлетворяют следуюшим условиям:

1) $R_{n_{j}^{\prime}, m_{j}}=E_{n_{j}^{\prime}}, j=0,1,2, \ldots, k$;

2) $E_{n_{k}^{\prime}}<\cdots<E_{n_{1}^{\prime}}<E_{n_{0}^{\prime}}$.

Для этого в качестве $n_{0}^{\prime}$ возьмем наименьшее число $n_{0}^{\prime} \geqslant 0$, для которого $R_{n_{0}^{\prime}, m_{0}}=$ $E_{n_{0}^{\prime}}$. Ясно, что $n_{0}^{\prime} \leqslant n_{0}$. Если $n_{0}^{\prime}>0$, то можно считать, что $E_{n_{0}^{\prime}}<E_{n_{0}^{\prime}-1}$. В противном случае

$$
R_{n_{0}^{\prime}, m_{0}}=E_{n_{0}^{\prime}}=E_{n_{0}^{\prime}-1} \leqslant R_{n_{0}^{\prime}-1, m_{0}} \leqslant E_{n_{0}^{\prime}-1}
$$

и $n_{0}^{\prime}$ можно заменить $n_{0}^{\prime}-1$. Определим теперь наименьшее $n_{1}^{\prime}>n_{0}^{\prime}$, для которого $R_{n_{1}^{\prime}, m_{1}}=E_{n_{1}^{\prime}}<E_{n_{0}^{\prime}}$. Из условий $(3)$ и (4) очевидно, что $n_{1}^{\prime} \leqslant n_{1}$ и можно считать, что $E_{n_{1}^{\prime}}<E_{n_{1}^{\prime}-1}$. В противном случае

$$
R_{n_{1}^{\prime}, m_{1}}=E_{n_{1}^{\prime}}=E_{n_{1}^{\prime}-1} \leqslant R_{n_{1}^{\prime}-1, m_{1}} \leqslant E_{n_{1}^{\prime}-1} .
$$

Отсюда следует, что

$$
R_{n_{1}^{\prime}-1, m_{1}}=E_{n_{1}^{\prime}-1}=E_{n_{1}^{\prime}}<E_{n_{0}^{\prime}}
$$

и $n_{1}^{\prime}$ можно заменить на $n_{1}^{\prime}-1$. Далее, по индукции определяются $n_{2}^{\prime}, n_{3}^{\prime}, \ldots, n_{k}^{\prime}$. Поскольку

$$
R_{n_{j}^{\prime}, m_{j}}=E_{n_{j}^{\prime}}<E_{n_{j}^{\prime}-1}, \quad j=0,1,2, \ldots, k,
$$

то на основании леммы получаем, что $n_{j+1}^{\prime} \geqslant n_{j}^{\prime}+m_{j}+1$. Теорема доказана.

Отметим, что близкие вопросы, относящиеся к теоремам Бернштейна и Долженко, затрагиваются также в работах [6], [9], [10].

В заключение автор выражает благодарность профессору А. А. Пекарскому за полезные обсуждения полученных результатов и внимание к работе.

\section{СПИСОК ЦИТИРОВАННОЙ ЛИТЕРАТУРЫ}

[1] Даугавет И. К. Введение в теорию приближения функций. Л.: ЛГУ, 1977.

[2] Долженко Е. П. Сравнение скоростей рациональной и полиномиальной аппроксимаций // Матем. заметки. 1967. Т. 1. № 3. С. 313-320.

[3] Boehm B. Functions, whose best rational Chebyshev approximations are polinomials // Numer. Math. 1964. V. 6. № 3. P. 235-242.

[4] Lorentz G., v. Golitschek M., Makavoz Y. Constructive Approximation. Advanced Problems. New York: Springer-Verlag, 1996.

[5] Пекарский А. А. О случаях совпадения наилучших равномерных полиномиальных и рациональных приближений // J. Technical University at Plovdiv. 1996. V. 2. "Fund. Sci. and Appl.". P. 41-44.

[6] Назаренко М. А. Некоторые свойства рациональных аппроксимаций. // Дисс. ... к.ф..-м.н. М.: МГУ, 1997.

[7] Ахиезер Н. И. Лекции по теории аппроксимации. М.: Наука, 1965.

[8] Гончар А. А. Обратные теоремы о наилучших приближениях на замкнутых множествах // Докл. АН СССР. 1959. Т. 128. № 1. С. 25-28.

[9] Вячеславов Н. С., Рамазанов А. К. О степени рациональных функций наилучшего приближения в $L_{p}\left(R^{m}\right) / /$ Матем. заметки. 1993. Т. 53. № 2. С. 37-45.

[10] Пекарский А. А. Существование функции с заданными наилучшими равномерными рациональными приближениями // Изв. АН Беларуси. Сер. физ. -матем. 1994. №1. С. 23-26.

Гомельский государственный 